\title{
“Knowledge I seek because culture doesn't work anymore ... It doesn't work, death comes": the experiences of third-generation female caregivers (gogos) in South Africa discussing sex, sexuality and HIV and AIDS with children in their care
}

Jane E. Simmonds ${ }^{1,2,3^{*}}$ (D), Charles D. H. Parry ${ }^{4,5}$, Fareed Abdullah ${ }^{1}$, Nadine Harker Burnhams $s^{4,6}$ and Nicola Christofides ${ }^{2}$

\begin{abstract}
Background: Sexual reproductive health communication between parents and children has been shown to promote safer sexual choices. In many South African households, third-generation female caregivers, often grandmothers or other older females, locally known as gogos, are primary caregivers of children due to parents being deceased or absent. Subsequently, the responsibility of talking about sex and related issues has shifted to these gogos. This study explored the experiences of gogos living in Alexandra, Johannesburg on talking about sex, sexuality and HIV and AIDS with children aged 10-18 years that are in their care.
\end{abstract}

Methods: Ten primary caregivers were purposively selected. Data were collected through in-depth individual interviews. Thematic analysis was performed and inductive codes and themes identified.

Results: All gogos selected found it difficult to discuss sex, sexuality and HIV and AIDS due to culture and traditional values impacting on personal experiences as well as generation and gender barriers. Perceived low selfefficacy due to low levels of knowledge and limited skills in speaking about sex, sexuality and HIV and AIDS also contributed to low levels of sexual reproductive health communication.

Conclusions: This study highlights the need for interventions that focus on improving gogos' knowledge about sexual reproductive health in addition to providing them with the skills to talk about sex, sexuality and HIV and AIDS with children in their care.

Keywords: HIV, AIDS, Sexual communication, Grandmothers, Third-generation caregivers, South Africa, Sexual reproductive health, Sex

\footnotetext{
* Correspondence: jane.simmonds@mrc.ac.za

'Office of AIDS and TB, South African Medical Research Council, Pretoria, South Africa

${ }^{2}$ School of Public Health, University of the Witwatersrand, Johannesburg, South Africa

Full list of author information is available at the end of the article
}

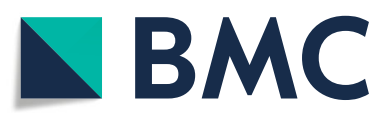

(๑) The Author(s). 2021 Open Access This article is licensed under a Creative Commons Attribution 4.0 International License, which permits use, sharing, adaptation, distribution and reproduction in any medium or format, as long as you give appropriate credit to the original author(s) and the source, provide a link to the Creative Commons licence, and indicate if changes were made. The images or other third party material in this article are included in the article's Creative Commons licence, unless indicated otherwise in a credit line to the material. If material is not included in the article's Creative Commons licence and your intended use is not permitted by statutory regulation or exceeds the permitted use, you will need to obtain permission directly from the copyright holder. To view a copy of this licence, visit http://creativecommons.org/licenses/by/4.0/ The Creative Commons Public Domain Dedication waiver (http://creativecommons.org/publicdomain/zero/1.0/) applies to the data made available in this article, unless otherwise stated in a credit line to the data. 


\section{Background}

Despite the declining HIV and AIDS prevalence globally, nearly half of all new HIV infections still occur among youth, especially in sub-Saharan Africa [1]. In South Africa, of the 231,000 new HIV infections in 2017, 38\% were among persons aged 15-24 years, with around 66, 000 of these new infections in girls and young women [2]. Adolescents are vulnerable to HIV infection as adolescence is a time of experimentation with sex [3] and when sexuality and sexual behaviour is shaped [4]. Adolescent sexual decision-making and behaviour are influenced by a number of factors at individual, peer, family, community and societal levels, with parents in particular playing a significant role [5]. Interventions that encourage the development of healthy sexual norms among adolescents can lead to a reduction in risk taking behaviour [4]. Research on the role of communication between parents and children in the development of adolescent sexual behaviour has established the importance of parent-child sexuality communication in reducing sexual risk behaviour [5-8].

Programmes to promote sexual communication with adolescents tend to focus on adolescents and/or mothers and sometimes both parents [5]. Globally, however, older people, usually older women and often grandmothers, are increasingly facing the challenges of raising children [9, 10]. In South Africa, many older women have become the primary caregivers of children orphaned by AIDS [11-13] and parents being absent from the home due to migration to other provinces in seek of work, job seeking, ill health or incarceration [10]. In 2018, it was estimated that nearly 4 million children in South Africa were living with a grandparent or an aunt [14]. There are a number of challenges facing these older caregivers which require new parenting strategies relevant to them, their experiences and their societies [10]. Among the challenges faced by these grandparents is the important issue of sexual and reproductive health (SRH) communication with the grandchildren in their care [15].

There is little available research globally on the role of grandmothers in SRH and the effectiveness of the grandparent-child dyad relationship in relation to SRH communication [16, 17]. In the United States, AfricanAmerican grandparent caregivers felt unprepared to discuss topics regarding sexuality and sexual health with their grandchildren [18] and they required assistance communicating with their grandchildren about SRH because they belonged to a generation that found SRH topics embarrassing to discuss [19]. In spite of barriers to talking about sex, grandmothers wanted to have open and informed SRH conversations with their grandchildren $[16,17]$. Parent-child conversations about SRH placed value on sexual abstinence as opposed to grandparentchild SRH conversations which addressed sex and sexuality [17].
In sub-Saharan Africa, interventions implemented in West and Southern Africa found that grandmothers can play an important role in SRH behaviour and choices of children in their care [20-22]. In a Ghanaian project, grandmothers served as important resources for reducing HIV infection and unintended pregnancy in adolescents. Both grandmothers and adolescents experienced the project's intergenerational activities as beneficial [20]. In Malawi, trained "agogos" (the local name for grandmothers) helped young girls acquire sexual health information [21]. Both the trained "agogos", and the girls they counselled, were more comfortable with SRH communication and were more likely to have interactive SRH discussions [21]. Meanwhile, in Botswana, grandmothers raising HIV-positive children found SRH conversations with these children very difficult due to cultural beliefs and that programmes to support these conversations between grandmothers and children were needed to address the needs of grandmothers in particular [22].

In South Africa, there is some research on the role of caregivers, who are not always biological parents, and how they communicate with children in their care about $\mathrm{SRH}$ issues [23, 24]. Grandparents in the Eastern Cape were unsuccessful in communicating SRH matters to the children they were raising and they needed to be supported in SRH communication with their grandchildren [25]. An evaluation of a family-centred adolescent HIV prevention programme, Let's Talk, to address individual HIV transmission risk factors common among orphaned and vulnerable adolescents where nearly $50 \%$ of caregivers were grandmothers or aunts, found statistically significant improvements in adolescents' HIV and condom use knowledge, as well as condom negotiation selfefficacy [26]. In addition, communication about healthy sexuality between caregiver and adolescent also improved [26].

Although there is limited research in South Africa on grandmothers and SRH communication with the children they are raising, there are studies that address the role of grandmothers in homes. Maternal grandmother involvement in adolescent adjustment was associated with more prosocial behaviour and less internalising of problems in adolescents in third-generation households [27]. Grandparent involvement in the emotional and behavioural health of adolescents was identified as a resource for promoting social and emotional competence in adolescents [28].

Literature on third-generation caregivers speaks to experiences related to age and frailty which are unique features of third-generation caring $[13,15]$. Generational differences present their own specific challenges; for example child discipline and disharmony in the family in intergenerational relationships [13]. As a result, grandparents may not feel prepared to conduct SRH conversations, and this is 
exacerbated by the lack of resources to assist and prepare grandparents for SRH conversations [15, 22].

In this paper we document the experiences of older women, including grandmothers and aunts, referred to as gogos, in Alexandra, Johannesburg, of speaking to children aged 10 to 18 years in their care about sex, sexuality and HIV and AIDS. The findings are from interviews conducted as part of an evaluation of a skills-building workshop on SRH that aimed to improve gogos SRH communication skills. For the purposes of this paper, 'grandmothers' and 'gogos' refer to "experienced, senior women in the household who are knowledgeable about all matters related to the health, development and wellbeing of children and their mothers. This includes not only maternal and paternal grandmothers, but also aunts and other older women" [29]. Although there was a range in ages of the older caregivers from 52 to 77 years, the women all self-identified as "gogos". The terms "grandchild/grandchildren/children" relate to all children and adolescents being raised by a gogo, aged 10-18 years and therefore not necessarily just biological grandchildren.

The purpose of this paper is to describe and explore the gogos' experiences and perceptions of talking about sex, sexuality and HIV and AIDS with the children they are raising.

\section{Methods}

\section{Participants and study site}

The qualitative study took place between February 2014 and January 2015. Gogos living in Alexandra, Johannesburg were recruited for the study by the researcher (JS) through a meeting held at a local non-profit organization (NPO). Alexandra is a crowded, low-income suburb with high levels of unemployment, poverty and crime. The women were selected because they were the primary caregivers of children between the ages of 10 and 18. Some gogos had other children who they were caring for outside of the specified age group where the household included children younger than 10 and older than 18 years but these dependents were not the focus of the SRH communication. All the women self-identified as gogos regardless of their age or whether they were raising biological grandchildren or not. They all attended weekly GOGO Days at Ratang Bana, a community-based NPO that cares for around 600 vulnerable children from the community. The GOGO Days were established primarily for attendees to work on income generation projects like crafting and vegetable growing.

We purposively selected ten gogos or older female caregivers who met the criteria of raising children aged 10-18. The reason for selecting this number of participants was to collect rich data at multiple timepoints including two rounds of in-depth interviews and focus group discussions. The gogos spoke and understood English as the intervention being evaluated was a training workshop conducted in English using a resource book, $H I V \mathcal{E} A I D S$ which was available in English [30]. The selected gogos were the primary caregivers of boys and/or girls within the specified age group. The women who were recruited were not all biological grandmothers of the children for whom they were caring.

\section{Data collection}

Data were collected through in-depth individual interviews conducted in either Setswana or isiZulu by a trained research assistant. Pseudonyms were used in all transcripts and when reporting the findings. The research assistant was trained by the lead researcher (JES) on the purpose of the research study the data collection methods. The head of Ratang Bana in Alexandra granted permission to recruit participants for the study and informed consent was obtained from each gogo who expressed an interest in participating in the study. A separate consent form was used to get permission for creating audio-recordings of the interviews. The Human Research Ethics Committee at the University of the Witwatersrand approved the research study.

Interviews were held in either a room on the premises of Ratang Bana, which was private and designated for counselling at the centre, or at the homes of the individual participants depending on their preference. Basic sociodemographic information such as age, education, number of children in their care, age and sex of grand children being raised were collected, as well as sources of income including whether they were recipients of foster-care grants, old age "pensions" or other streams of income.

The interviews were guided by an interview guide with a scripted introduction, which was read at the beginning of each in-depth interview (See Additional File 1). The guide consisted of open-ended, non-directive questions, moving from the general to the specific. The interviewer started by asking about the gogos' relationships with the children in their care in terms of activities, needs and demands, and whether there were problems experienced looking after the children. The interviewer then proceeded to questions specifically focusing on feelings about talking about sex, sexuality and HIV and AIDS; who should speak to children about these topics; what would make it easier to have these conversations; and what difficulties were experienced when talking about sex, sexuality and HIV and AIDS. The gogos were also asked about the last time they had talked to children in their care about sex, sexuality and HIV and AIDS, and if they had never spoken to their grandchildren about these topics the reasons why they had not. Interviews lasted for approximately $1 \mathrm{~h}$.

\section{Data analysis}

Audio recordings of the interviews were translated and transcribed verbatim. Identifying information was removed 
and pseudonyms used. Transcripts were imported into MAXQDA 10 for data coding and analysis. The thematic analysis was inductive, and codes and themes were developed after a close reading of the transcripts [31]. Text segments from the transcripts were assigned codes based on the ideas or categories being discussed. A code schema was developed with definitions for each code to facilitate further coding. The senior co-author (NC) reviewed the codes and definitions of each code and how they were applied to a transcript. Any ambiguity was discussed and clarified. Memos were written throughout the process of coding which tracked the patterns and co-occurring themes [31]. Mind maps were used to visualise the themes which were organised into patterns. These patterns were then reviewed and interpreted. Relevant salient quotes for each emergent theme were selected to support each theme during the process of writing up.

\section{Results}

Socio demographic characteristics of participants

Although all the older women in the sample selfidentified as gogos, only two of the ten women were biological grandmothers and the others were aunts, great aunts, foster mothers, an adoptive mother and a caring older woman from the community. They cared for a combination of biological grandchildren, biologicallyrelated children and other third-generation children. The average age of the women who participated in the study was 60.3 years, with a range from 52 to 77 years (Table 1). The average number of children being cared for by each gogo was three. There were 18 male and 18 female children being cared for by the gogos in the study.
The average age of the child dependents in their care was 13.5 years with a range from 2 to 21 years, although the SRH communication focused on children between 10 and 18 years. None of the gogos were formally employed. Their sources of income were mainly social security grants and a few had some form of selfgenerated income. This self-generated income took the form of hawking, rentals and charitable support.

Experiences of the gogos talking about sex, sexuality and HIV and AIDS with their grandchildren

Various themes emerged regarding the gogos' feelings and perceptions regarding talking about sex, sexuality and HIV and AIDS with their grandchildren. These included personal, contextual and structural barriers. On a personal level, barriers between gogos and grandchildren about talking about sex, sexuality and HIV and AIDS included the personal difficulties experienced by gogos due to past experiences with talking about sex, a lack of skills and knowledge of how to have these conversations, and a fear that talking about sex would encourage sexual behaviour. Contextual and structural barriers housed in cultural beliefs, gender and generational differences also affected the feelings and experiences of these gogos related to talking about sex, sexuality and HIV and AIDS.

\section{Personal barriers experienced by gogos in talking to grandchildren about sex}

Difficulty talking about sex, sexuality and HIV and AIDS

All the participants expressed negative feelings regarding talking about sex, sexuality and HIV and AIDS and

Table 1 Socio-demographic characteristics of gogos

\begin{tabular}{|c|c|c|c|c|c|}
\hline Pseudonym & Age & Number of dependants & $\begin{array}{l}\text { Ages of children in } \\
\text { in gogos care }\end{array}$ & Source of income & $\begin{array}{l}\text { Total monthly income disclosed } \\
\text { in interview }\end{array}$ \\
\hline Thoko & $60-65$ & 1 & $10-15$ & $\begin{array}{l}\text { Senior Citizen grant } \\
\text { Foster Care Grant }\end{array}$ & R2150 \\
\hline Thuli & $60-65$ & 3 & $\begin{array}{l}15-20(2) \\
20-25\end{array}$ & $\begin{array}{l}\text { Hawking } \\
\text { Foster Care Grant }\end{array}$ & R840 \\
\hline Bontle & $50-55$ & 2 & $0-5,15-20$ & Social Welfare Grant & R320 \\
\hline Sindi & $55-60$ & 4 & $\begin{array}{l}0-5(2) \\
5-10,15-20\end{array}$ & $\begin{array}{l}\text { Rent Income } \\
\text { Social Welfare Grant }\end{array}$ & R320 \\
\hline Florence & $70-75$ & 2 & $15-20,20-25$ & $\begin{array}{l}\text { Senior Citizen Grant } \\
\text { Social Welfare Grant }\end{array}$ & $\mathrm{R} 1670$ \\
\hline Bongi & $60-65$ & 2 & $0-5,15-20$ & Not disclosed & - \\
\hline Lerato & $50-60$ & 2 & $10-15,15-20$ & $\begin{array}{l}\text { Social Welfare Grant } \\
\text { Help from local NGO }\end{array}$ & R320 \\
\hline Agnes & $50-60$ & 3 & $\begin{array}{l}10-15(2) \\
15-20\end{array}$ & $\begin{array}{l}\text { Social Welfare Grant } \\
\text { Monthly Stipend from Projects }\end{array}$ & R820 \\
\hline Mercy & $50-55$ & 2 & $10-15(2)$ & Foster Care Grant & $\mathrm{R} 840$ \\
\hline Faith & $70-89$ & 2 & $10-15,20-25$ & $\begin{array}{l}\text { Foster Care Grant } \\
\text { Senior Citizen Grant }\end{array}$ & R2190 \\
\hline
\end{tabular}


commented on the difficulty of speaking to their grandchildren about these issues:

"Hey lady ..., that's going to be difficult. That is going to be really difficult." - Sindi (55-59yrs, caregiver of four children)

Although all the participants found talking about sex difficult, there was an acceptance or understanding amongst the participants that "how things are today is nothing like they used to be" (Bongi) and that there is now a need to talk to children about SRH. Lerato talked about trying to discuss issues that were not spoken about to her when she was growing up and how difficult this made talking about HIV and AIDS, and sex and sexuality.

Several gogos spoke about their lack of experience, knowledge and skills on this topic:

"The problem is that ... I would not know what to say." - Bongi (60-64yrs, caregiver of two children).

Some participants felt that, despite the fact that today's generation is far better informed than they were, knowing, for example, that babies come from the "stomach", and that the topic of sex does get covered at school, sex was still a very difficult topic for them to discuss. Reasons for this included cultural taboos, lack of SRH communication experiences in their own adolescence and general lack of information.

“Now you must discuss issues [that] weren't discussed with you and therefore aren't open about is a bit difficult ... When the time to discuss it all of these topics ... you feel overwhelmed and somewhat defeated." - Lerato (55-59yrs, caregiver of two children)

\section{Past experiences of talking about sex, sexuality, HIV and AIDS}

A number of participants mentioned feelings of fear and experiences of corporal punishment at the hands of their parents linked to their own sexual experiences growing up. Florence described the way her parents used fear and threats when speaking about sex when she was growing up. She was also told falsehoods about where babies came from: "in suitcases delivered by nurses". Shame was also mention as a reason to not talk about SRH:

"We are still ashamed to discuss certain issues. Sex this and that ... It is difficult finding ways to tackling these issues as we never had such conversations nor was it ever taught to us at school." - Sindi (55-59yrs, caregiver of four children)
Fear of talking about sex, sexuality, HIV and AIDS

Another source of anxiety raised by one gogo was based on the belief that they had grown up with that talking about sex sexuality, HIV and AIDS would encourage sexual activity.

"You see in our times, our parents felt if you taught a child about sex you were promoting the idea." Sindi (55-59yrs, caregiver of four children)

Sindi recounted that although she had spoken to her own children about sex in the past, she voiced her frustration at the fact that her children had had unplanned pregnancies in spite of the fact that she had spoken to them. She now regarded these past conversations about SRH as unsuccessful as it "failed us, because now we have these children [grandchildren] in the yard". She expressed disappointment that her children had had babies in spite of her attempts to prevent this by speaking to them and "warning" them.

\section{Contextual and structural barriers to talking about sex, sexuality and HIV and AIDS encountered by gogos}

Contextual and structural barriers played a significant role in how effective gogos were in their communication with their grandchildren about sex and HIV and AIDS. Although contextual and structural, these barriers were informed and defined by the personal experiences of the participants and through their perceptions of culture, gender, age and the generation gap.

\section{Cultural barriers}

A number of gogos brought up cultural issues as a barrier to talking about sex, sexuality and HIV and AIDS with their grandchildren. Communication about sex was not something participants had experienced during their own adolescence, due to the belief that parents discussing sex with their children would encourage or enable sexual activity and the fact that HIV and AIDS did not exist in their youth.

Although Sindi explained "in our culture, we are not used to that" and "it was not easy when it came to my children", she felt very strongly that cultural beliefs restricting the sharing of open and factual information on SRH needed to be rejected and that gogos needed to move beyond cultural norms and address these issues with their grandchildren in order to save lives.

“There isn't a culture that I'm committed to, to the point where I would say, I don't do this, I don't do that, because of my culture. Knowledge I do seek because culture doesn't work anymore ... It doesn't work, death comes." - Sindi (55-59 yrs, caregiver of four children). 


\section{Gender}

Participants raised the issue of gender norms when speaking to grandchildren about sex, sexuality and HIV and AIDS. Not only did these gender norms apply to the gogos and who they spoke to, they also related to the gogos understanding of adolescent sexuality. Sindi explained how "[they] are used to boy children being spoken to by a man", while one participant described how, when she tried to bring up the subject, her grandson got "uncomfortable" and tried to close down the conversation as soon as possible. In addition, these gogos felt that their already limited knowledge was particularly lacking when it came to speaking to their grandsons about issues pertaining to males. Sindi claimed boys would "puzzle" her, as she had no experience with them: "honestly I don't know what I would say to a boy," she said. Boys were advised to go for HIV testing which was a strategy for gogos to facilitate them getting information and advice from a trusted source.

"It is important to check, as you [a boy in her care] may not always know what the girl gets up to ... so you should go and check [get tested] frequently." Bongi (60-65ys, caregiver of two children).

When talking to girls, gogos frequently counselled them to avoid boys as much as possible in order to protect themselves. Gogos regarded boys as being a threat to girls. The majority of gogos perceived boys as having only one agenda with regards to girls and that was to have sex. Sex with boys was communicated to girls as something that would result in sexually transmitted infections and pregnancy.

"I need you to take good care of yourself and keep clear of boys when you play. When you play, play with girls." - Thoko (60-64yrs, caregiver of one child).

\section{Generation barrier}

Several gogos spoke about the generation gap between themselves and their grandchildren and the need for increased understanding between them.

"There is a gap between us that needs to be bridged. They need to learn how to speak to us so we can understand one another." - Patience (55-59yrs, caregiver of three children)

Respect for the older generation, and the fear of this generation losing respect, were themes that emerged during the interviews. Agnes explained that she feared she would lose the respect of her grandchildren if she spoke to them about sex, sexuality and HIV and AIDS and, having lost respect for her, her grandchildren would be more likely to participate in sexual activity.

"If I speak to them about such issues ... they will end up taking you for granted. Isn't it 'Gogo can speak to me about this so ...' they'll overstep their boundary" - Agnes (55-59yrs, caregiver of three children)

Sindi suspected some of her grandchildren's responses, which included laughter, could have been due to the fact she was "gogo" and that they were possibly embarrassed by this: "Since it came with "gogo", they would laugh when they see some of the things." Sindi also mentioned that the issue of age and the generation gap as a possible reason for difficulty talking about sex, sexuality and HIV and AIDS with her grandchildren. She explained that her grandchild was not as close to her in age as he would have been to his mother.

"That is going to be really difficult, especially when they are not your [biological child]. There is no way of going beyond the fact that I'm not his mother." Sindi (55-59yrs, caregiver of four children)

Sindi and Lerato both spoke about the difficulties of trying to get their grandchildren to not only listen to their advice but also to subsequently follow this advice, rather than being influenced by other factors such as the behaviour of their peers.

"For me, it is not difficult to talk to my kids, but our kids do not want to listen. You can tell him or her something here in the house but when he is going outside to the friends, they teach their way of living."

- Sindi (55-59yrs, caregiver of four children)

\section{Discussion}

All gogos in the study provided care and parenting to the children they looked after and expressed a common desire to keep them safe and healthy. Despite acknowledging the need to speak to children aged 10-18 years in their care about sex, sexuality and HIV and AIDS, the experiences of the gogos in doing so were extremely limited and fraught with challenges and fears. The difficulties were exacerbated by contextual and structural barriers such as culture, gender and generational issues, as well as personal challenges which included past experiences of SRH conversations and a lack of skills and knowledge around SRH. As a result of these difficulties most of the gogos had never had SRH conversations with the 10-18 year old children in their care.

Culturally, SRH communication in Africa is difficult, as many communities regard conversations around 
puberty, sex and sexuality as taboo [5]. Traditional values and cultural norms were seen by the gogos as barriers to open conversations about sex, particularly as many gogos had very limited experiences of these types of conversations while growing up. When, or if these conversations did occur, they were impacted by normative beliefs and the culture of their childhood and often contained misinformation. All the gogos spoke about the complicated and difficult SRH conversations that they had had both with their own parents and while at school. Together with past experiences of SRH conversations, gender roles and norms and generation issues appeared to impact gogos' perceptions of how to communicate about SRH translating into challenges and barriers in SRH communication between the gogos and the third-generation children they were raising.

Research on parent-child SRH communications has found that the genders of both the parent and the child appear to play a dominant role for both parties as to the manner in which SRH discussions occurred, as women tend to speak to girls and men tend to speak to boys $[5,8]$. The gogos in this study did not express preferences related to which gender child they spoke to, but they did comment on the fact that it was the social norm for men to speak to boys and for women to speak to girls, and they found it more difficult to speak to boys. In addition, the content of the conversations was different when speaking to boys and girls. Messages to girls often contained warnings to keep away from boys and to control their sexuality which ties in to broader gender norms about how girls should behave.

Several of the participants commented on the fact that they were gogos and not the mothers of these grandchildren. Generational differences presented their own specific challenges to grandparents who did not feel prepared to deal with SRH conversations [15]. As reported in a separate study by Nyasani et al. [13], the gogos experienced aspects of intergenerational conflict and problems with child discipline and disharmony. These added to the problems and barriers around SRH communication between gogos and their grandchildren. Gogos experienced perceived dismissal from grandchildren, feared losing respect in the eyes of their grandchildren and being laughed at by their grandchildren when attempting to initiate SRH communication.

In addition to the cultural and social obstacles discussed above, the gogos explanation for the lack of SRH communication in their youth was that their parents feared that discussion and knowledge of sexual topics would lead to children engaging in sexual activities. In addition, the conversations about sex that the gogos had had with their own parents had often been negative and punitive. Parental involvement in sexual communication is generally a new notion for South African families [23] and many of the gogos in the parental role found themselves having the same types of negative conversations about SRH when speaking to grandchildren about sex, sexuality and HIV and AIDS as they had had with their own children.

The fear that speaking to children about sexual behaviour would promote sexual activity is common theme in sub-Saharan Africa [5]. The gogos explained that their parents shared this fear and it was at least partly responsible for the lack of perceived efficacy on the part of the gogos in addressing SRH with their own children. Many gogos reported limited skills or experience in how to have conversations about sex, sexuality and HIV. A study by Cornelius et al. [18] found that African American grandmothers did not fear that talking about sex would promote sexual behaviour in their grandchildren, and embraced the idea of talking to their grandchildren about sex [18]. The results of our study aligns with Cornelius et al. [18] as fear of promoting sexual activity was not mentioned by the gogos as a barrier to having SRH conversations their grandchildren or children in their care. The gogos in this study showed that there has been a shift away from this belief based on their lived experiences that not talking sex, sexuality and HIV does not prevent sexual behaviour or HIV infection. In fact, many of them felt that SRH conversations were necessary.

Personal beliefs and experiences cannot be totally discounted as a barrier to SRH discussions, as many parents are from a generation where discussing sexuality topics was linked to embarrassment $[5,8]$. Although grandparents can be receptive to discussing sex and sexuality with their grandchildren, they experience different levels of comfort with these conversations [18]. In our study gogos expressed discomfort and a lack of confidence and ability to acknowledge, confront or discuss the difficult and uncomfortable topic and issues of adolescent sexual behavior and felt they lacked the skills to do this although this could potentially be mitigated by providing support for the gogos, enabling them to conduct the discussions more effectively.

Despite increasing evidence of programmes targeting grandmothers to support SRH communication having positive outcomes $[20,21]$, only one gogo in our study had received any prior HIV training. A South African study in the Eastern Cape reported that grandparents were unsuccessful in communicating SRH matters to youth and concluded that grandparents should be supported in SRH conversations with their grandchildren [25]. A similar need was identified through our research, with the participants acknowledging the value of these conversations in reducing HIV infection and unintended pregnancy. Our findings clearly demonstrate the need to engage with gogos to increase effective SRH communications as gogos 
stated that SRH conversations would be easier if they, and the children in their care, had access to workshops which built communication skills on these topics.

A number of studies in South Africa have commented on the importance of the role of extended family and caregivers other than parents [23, 24, 26-28] and this study reinforces the view that it is essential to examine the changes in family composition in SRH conversations in South Africa [23, 26-28]. In addition, this study supported findings in other research $[17,22]$ for the need for healthcare providers and programmes to recognise that there are differences in SRH communication and conversations between parents and grandparents. Aubel (2005) showed that the concern that grandmothers might be biased and unable to adapt to new ideas is incorrect and that this should not be a reason that interventions do not make use of older people as drivers of community and family-based interventions [29]. Grandmothers are in fact open and receptive to change and new ideas and can be very powerful resources in promoting health changes [29]. Like Aubel (2005), our findings challenged the stereotype of grandmothers as being conservative and resistant to change as all the gogos expressed a desire to be able to have informed and effective SRH conversations with the grandchildren they were looking after or raising.

The findings need to be considered in the light of the following limitations related to the collection of data and the role of the lead researcher. She had prior links to Ratang Bana which could have influenced her interpretation of the findings and the data analysis because she was aware of the difficulties experienced by the participants. In addition, the researcher was sympathetic to parenting issues as a mother of adolescent children herself and had established relationships with some of the participants prior to the commencement of the research. Furthermore, although the participants were typical of gogos in Alexandra, they were all accessing support at a small, local NGO which could have influenced their experiences and how they engaged in the interviews. In addition, only a small number [10] of older women met the criteria to participate in this study due to the NGO being a small community organisation. Saturation was reached on several topics vindicating the decision to go with a relatively small number of study participants. However, some of these limitations were mitigated by having an experienced interviewer who did not have any prior connection with the organisation, spoke the same languages and was a similar age to participants.

Another limitation in this study is that the ages of the children being referred to in the gogos' interviews was not recorded and it is indeed possible that SRH conversations were easier with older children (15-17 years of age) than younger children (10-14 years). Furthermore, it is not possible in some instances to know if the gogos were referring to boys or girls in their comments. It is likely that the experiences of SRH communication for gogos differ across ages and genders so future research should look more carefully at this.

\section{Conclusion}

Talking about sex is not easy for gogos due to personal and contextual and structural barriers. Personal barriers for the gogo included the difficulty and fear of talking about SRH and their own experiences growing up of not talking about sex, sexuality and HIV and AIDS. Contextual and structural barriers to talking about sex, sexuality and HIV and AIDS included cultural beliefs and practices, gender issues and the generation gap experienced between the gogos and the children they were raising. Many grandmothers and older female members in the community, the gogos, are primary caregivers of children and are fulfilling parental roles in raising children. This parental role needs to be recognised and issues affecting gogos need to be acknowledged. Gogos need to become part of, if not particularly targeted by, interventions to improve the SRH outcomes of the children they are raising.

\section{Abbreviations}

AIDS: Acquired Immune Deficiency Syndrome; HIV: Human ImmunoDeficiency Virus; NC: Nicola Christofides; SHR: Sexual Reproductive Health

\section{Supplementary Information}

The online version contains supplementary material available at https://doi. org/10.1186/s12889-021-10494-5.

Additional file 1. Communication between grandmothers and their grandchildren. Interview Guide 1 (for in depth interview before skills training workshop).

\section{Acknowledgements}

Thank you to the gogos of Ratang Bana for your time and for sharing your thoughts, feelings, and experiences.

\section{Authors' contributions}

JES conducted the research under the supervision of NC. CDHP, FA, NHB and NC contributed to the writing, analysis and preparation of the manuscript. All authors have read and approved the final manuscript.

\section{Funding}

The design of the study, the collection, analysis and interpretation of data for the study was self-funded by JE Simmonds. JE Simmonds, CDH Parry, F Abdullah and $\mathrm{N}$ Harker Burnhams are employed by the South African Medical Research Council. N Christofides is employed by the School of Public Health at the University of the Witwatersrand.

Availability of data and materials

Data and material can be requested from the corresponding author.

\section{Declarations}

Ethics approval and consent to participate

Ethics approval to conduct and report the study was given by The Human Research Ethics Committee at the University of the Witwatersrand and written consent was given by participants prior to participation. 


\section{Consent for publication}

Not applicable.

\section{Competing interests}

No potential conflict of interest was reported by the authors.

\section{Author details}

'Office of AIDS and TB, South African Medical Research Council, Pretoria, South Africa. ${ }^{2}$ School of Public Health, University of the Witwatersrand, Johannesburg, South Africa. ${ }^{3}$ Alcohol, Tobacco and Other Drug Research Unit, South African Medical Research Council, Pretoria, South Africa. ${ }^{4}$ Alcohol, Tobacco and Other Drug Research Unit, South African Medical Research Council, Cape Town, South Africa. ${ }^{5}$ Department of Psychiatry, Stellenbosch University, Tygerberg, South Africa. ${ }^{6}$ School of Public Health and Family Medicine, University of Cape Town, Cape Town, South Africa.

Received: 5 May 2020 Accepted: 24 February 2021

Published online: 09 March 2021

\section{References}

1. Kangmennaang J, Mkandawire P, Luginaah I. Determinants of risky sexual behaviours among adolescents in Central African Republic, Eswatini and Ghana: evidence from multi-indicator cluster surveys. Afr J AIDS Res. 2019; 18(1):38-50.

2. South African National AIDS Council South Africa's National Strategic Plan for HIV, TB and STIs 2017-2022. South African National AIDS Council; 2017.

3. Jaffe M. Adolescence: Chichester John Wiley; 1998.

4. Selikow T-A, Ahmed N, Flisher AJ, Mathews C, Mukoma W. I am not 'umqwayito': A qualitative study of peer pressure and sexual risk behaviour among young adolescents in Cape Town, South Africa. Scand J Public Health. 2009;37(2, Suppl):107-12.

5. Bastien S, Kajula L, Muhwezi W. A review of studies of parent-child communication about sexuality and HIV/AIDS in sub-Saharan Africa. Reprod Health. 2011;8(1):25-

6. Akers $\mathrm{AY}$, Holland $\mathrm{CL}$, Bost J. Interventions to improve parental communication about sex: a systematic review. Pediatrics. 2011;127(3):494510.

7. Santa Maria D, Markham C, Bluethmann S, Mullen PD. Parent-based adolescent sexual health interventions and effect on communication outcomes: a systematic review and meta-analyses. Perspect Sex Reprod Health. 2015:47(1):37-50.

8. Dilorio C, Pluhar E, Belcher L. Parent-child communication about sexuality: a review of the literature from 1980-2002. J HIV/AIDS Prev Educ Adolesc Children. 2003:5(3-4):7-32.

9. Hayslip B, Kaminski PL. Grandparents raising their grandchildren: a review of the literature and suggestions for practice. The Gerontologist. 2005:45(2): 262-9.

10. Mtshali MNG. The relationship between grandparents and their grandchildren in the black families in South Africa. J Comp Fam Stud. 2015; 46(1):75-83.

11. Cluver L, Operario D, Gardner F. Parental illness, caregiving factors and psychological distress among children orphaned by acquired immune deficiency syndrome (AIDS) in South Africa. Vulnerable Children Youth Stud. 2009:4(3):185-98.

12. Makiwane $M$, Kwizera SA. An investigation of quality of life of the elderly in South Africa, with specific reference to Mpumalanga Province. Appl Res Qual Life. 2006;1(3-4):297-313.

13. Nyasani E, Sterberg E, Smith H. Fostering children affected by AIDS in Richards Bay, South Africa: a qualitative study of grandparents' experiences. Afr J AIDS Res. 2009;8(2):181-92.

14. Hall K, Richter L, Mokomane Z. Lake L south African child gauge 2018. Cape Town: Children's Institute, University of Cape Town; 2018.

15. Brown EJ, Jemmott LS, Outlaw FH, Wilson G, Howard M, Curtis S. African American grandmothers' perceptions of caregiver concerns associated with rearing adolescent grandchildren. Arch Psychiatr Nurs. 2000;14(2):73-80.

16. Cornelius JB, LeGrand S, Jemmott L. African American grand families' attitudes and feelings and sexual communications: focus group findings. AIDS Care. 2009;20(2):133-40.

17. Cornelius JB, Xiong PH. Generational differences in the sexual communication process of African American grandparent and parent caregivers of adolescents. J Special Pediatr Nurs. 2015;20(3):203-9.
18. Cornelius JB, LeGrand S, Jemmott L. African American Grandparents'and adolescent Grandchildren's sexuality communication. J Fam Nurs. 2008; 14(3):333-46.

19. Cornelius JB, LeGrand S, Jemmott LS. African American grand families' attitudes and feelings about sexual communications: focus group findings. $J$ Assoc Nurs AIDS Care. 2009;20(2):133-40.

20. Aubel J. Rapid review of 'time with grandmas initiative. Accra: Government of Ghana, U.N. Population Fund; 2008.

21. Limaye RJ, Rimal RN, Mkandawire G, Kamath V. Tapping into traditional norms for preventing HIV and unintended pregnancy:harnessing the influence of grandmothers (Agogos) in Malawi. Int Q Commun Health Educ. 2015;36(1):53-70.

22. Shaibu S. Caring for HIV-positive orphans in the context of HIV and AIDS: perspectives of Botswana grandmothers. Int J Older People Nursing. 2016; 11(4):315-23.

23. Armistead L, Cook S, Skinner D, Toefy $Y$, Anthony ER, Zimmerman L, et al. Preliminary results from a family-based HIV prevention intervention for south African youth. Health Psychol. 2014;33(7):668-76.

24. Soon CN, Kaida A, Nkala B, Dietrich J, Cescon A, Gray G, et al. Adolescent experiences of HIV and sexual health communication with parents and caregivers in Soweto, South Africa. SAHARA-J. 2013;10(3-4):163-9.

25. Mangxola W. Grandparents' experience of communicating sexual matters to the youth: University of South Africa; 2007.

26. Thurman TR, Nice J, Luckett B, Visser M. Can family-centered programing mitigate HIV risk factors among orphaned and vulnerable adolescents? Results from a pilot study in South Africa. AIDS Care. 2018:30(9):1135-43.

27. Levetan J, Wild L. The implications of maternal grandmother coresidence and involvement for adolescent adjustment in South Africa. Int J Psychol. 2016;51(5):356-65.

28. Wild LG. Grandparental involvement and south African adolescents' emotional and behavioural health: a summary of research findings. Contemp Soc Sci. 2018;13(2):1-14.

29. Aubel J. Grandmothers: a learning institution. Washington, DC: United States Agency for International Development; 2005.

30. Appelbaum M. HIV \& AIDS. Jacana Media. 2009.

31. Hennink M, Hutter I, Bailey A. Qualitative research methods. Sage Publications; 2011

\section{Publisher's Note}

Springer Nature remains neutral with regard to jurisdictional claims in published maps and institutional affiliations.

Ready to submit your research? Choose BMC and benefit from:

- fast, convenient online submission

- thorough peer review by experienced researchers in your field

- rapid publication on acceptance

- support for research data, including large and complex data types

- gold Open Access which fosters wider collaboration and increased citations

- maximum visibility for your research: over $100 \mathrm{M}$ website views per year

At $\mathrm{BMC}$, research is always in progress.

Learn more biomedcentral.com/submissions 